\title{
A Day in the Life of Borderline Personality Disorder: A Preliminary Analysis of Within- Day Emotion Generation and Regulation
}

\author{
Matthew W. Southward, Stephen A. Semcho, Nicole E. Stumpp, Destiney L. MacLean, \& \\ Shannon Sauer-Zavala \\ Department of Psychology, University of Kentucky
}

\begin{abstract}
Author Note
Matthew W. Southward: https://orcid.org/0000-0002-5888-2769

Shannon Sauer-Zavala: https://orcid.org/0000-0002-7322-983X

This research was partially supported by grant K23MH106648 to Shannon Sauer-Zavala. Correspondence concerning this article should be addressed to Matthew W. Southward, Department of Psychology, University of Kentucky; 343 Waller Ave., Suite 303; Lexington, KY 40504. Phone: 859-562-1569. Email: southward@uky.edu
\end{abstract}

In press at Journal of Psychopathology and Behavioral Assessment https://doi.org/10.1007/s10862-020-09836-1 


\begin{abstract}
In Linehan's (1993) biosocial theory, borderline personality disorder (BPD) results in part from frequent, intense, negative emotions and maladaptive behavioral responses to those emotions. We conducted a secondary data analysis of an intensive single-case experimental design to explore hourly relations among behavioral responses and emotions in BPD. Eight participants with BPD $\left(M_{\text {age }}=21.57,63 \%\right.$ female; $63 \%$ Asian $)$ reported their emotions and behaviors hourly on two days. Participants reported a neutral-to-negative average emotional state with substantial variability each day. This emotional state was characterized most frequently by anxiety and joy. Participants tended to "dig into", or savor, experiences of joy, but problem-solve around, push away, or accept anxiety. Acceptance predicted hour-by-hour increases in negative emotion intensity, and pushing emotions away predicted hour-by-hour increases in positive emotion intensity. These results suggest that anxiety dominates the emotional experiences of people with BPD and co-occurs with a variety of emotion regulation strategies, while joy co-occurs with strategies designed to prolong emotional experiences. Despite its general adaptiveness, acceptance may be less effective, and pushing emotions away may be more effective, than other emotion regulation strategies at improving momentary negative emotions for those with BPD. We discuss the preliminary nature of these findings and encourage future researchers to build on them in larger samples with more severe presentations of BPD.
\end{abstract}

Keywords: borderline personality disorder, ecological momentary assessment, emotion, emotion regulation 


\section{A Day in the Life of Borderline Personality Disorder: A Preliminary Analysis of Within- Day Emotion Generation and Regulation}

Borderline Personality Disorder (BPD) is a severe psychiatric disorder characterized in part by affective instability, impulsive behaviors, and chronic suicidality (American Psychiatric Association [APA], 2013). This condition is estimated to affect approximately $1.6 \%$ of the general population (Torgersen, 2009) and up to $20 \%$ of psychiatric inpatients (Gunderson \& Links, 2008). Although there may be no gender differences in the rates of BPD in the general population (Grant et al., 2008), 75\% of people with BPD who present for treatment are women (Widiger \& Trull, 1993).

Linehan's (1993) biosocial theory, a leading conceptualization of BPD, posits that BPD “is primarily a disorder of the emotion regulation system" (Linehan, 1993, p. 43) and that emotion dysregulation stems from two precursors. First, people with BPD are characterized by a biological vulnerability to experience intense emotions (i.e., affective instability), which includes (a) greater reactivity to internal and external stimuli, (b) stronger emotional intensity, and (c) slower return to a baseline level of emotional arousal. Next, a childhood invalidating environment, in which emotional expressions are minimized, trivialized or punished by caregivers, reinforces the belief that emotions are dangerous and should be avoided. The transaction of these precursors, particularly among those with higher levels of trait impulsivity, is thought to lead to emotionally dysregulated behaviors characteristic of BPD (e.g., self-injurious behaviors, dysregulated eating, substance use; Chapman, 2019), designed to suppress or escape emotional experiences (Crowell et al., 2009).

Recent research has provided support for the centrality of affective instability and emotion dysregulation in BPD (Richetin et al., 2017; Southward \& Cheavens, 2018). Using 
behavioral laboratory tasks and retrospective self-report, researchers have found relatively consistent evidence that people with BPD, or likely BPD, demonstrate greater affective instability, especially in anger and anxiety, than healthy controls (Dick \& Suvak, 2018; EbnerPriemer, Kuo, et al., 2007) and clinical controls, including those with major depressive disorder, likely bipolar I disorder, bipolar II disorder, anorexia nervosa, other personality disorders (Nica \& Links, 2009; Reich et al., 2014; Rosenthal et al., 2008).1 BPD features have further been shown to be uniquely related to measures of affective instability in non-clinical samples (Cheavens \& Heiy, 2011; Tragesser \& Robinson, 2009).

\section{Daily Emotional Experiences in BPD}

However, studies designed to assess affective instability using laboratory-based and selfreport methods are limited in two important ways. First, these studies prioritize internal validity over external validity. Given that people with BPD tend to respond more strongly to personalized, idiographic stimuli than clinical or healthy controls (Kuo et al., 2014), laboratorybased and self-report studies may not be measuring the true intensity of responses as they occur in participants' daily lives. Second, these studies cannot capture the temporal unfolding of emotions over longer durations which is crucial to accurately capture the construct. One method researchers have used to address both of these limitations is ecological momentary assessment (EMA). In EMA studies, participants report on their experience multiple times throughout a study period using a mobile device to capture in vivo experiences. Because of these repeated, real-time assessments of experiences, EMA studies decrease the effects of recall bias and are better suited to track the temporal instability of symptoms characteristic of BPD (Santangelo et

1 Here, we use the phrases "likely" BPD and "likely" bipolar disorder because participants in these studies were not diagnosed with BPD or bipolar disorder but instead met thresholds on self-report questionnaires indicating the likely presence of these disorders. 
al., 2014). Several researchers have used EMA designs to assess real-time affective responses of people with BPD. People with BPD or elevated BPD features reported a greater number of and more intense negative emotions compared to both healthy controls (Berenson et al., 2011; EbnerPriemer et al., 2015; Ebner-Priemer, Welch, et al., 2007; Houben et al., 2018; Wolff et al., 2007) and those with depressive, anxiety, and psychotic disorders (Glaser et al., 2008; Stiglmayr et al., 2005; Trull et al., 2008).

Of note, EMA methods further allow the construct of affective instability (i.e., momentto-moment changes in emotional intensity, after removing temporal trends) to be distinguished from that of affective variability (i.e., within-person variability in emotional intensity; Trull et al., 2008). Researchers have shown that people with BPD report greater affective instability than those with depressive disorders regarding specific negative emotions (Trull et al., 2008) but not those with posttraumatic stress disorder or bulimia nervosa (Santangelo et al., 2014). Researchers have also shown that people with BPD report greater affective variability than those with depressive disorders (Trull et al., 2008) and healthy controls (Ebner-Priemer et al., 2015, cf. Cowdry et al., 1991).

\section{Behavioral Responses to Emotions in BPD}

In order to modulate negative emotional experiences, there is evidence that people with BPD overuse putatively maladaptive strategies relative to their use of adaptive strategies (Carpenter \& Trull, 2013; Kring \& Sloan, 2010; Neacsiu et al., 2013). Maladaptive emotion regulation strategies are conceptualized as those that contribute to greater long-term cognitive, behavioral, and/or social dysfunction when used repeatedly, whereas adaptive strategies are associated with improved functioning in these domains. Examples of maladaptive strategies include rumination, thought suppression, experiential avoidance, impulsivity, and self-harm or 
suicidal behaviors (Carpenter \& Trull, 2013). Adaptive strategies, on the other hand, include acceptance, cognitive reappraisal, and problem-solving (Aldao et al., 2010).

It should be noted that there is a burgeoning literature on the contextual factors that influence when and for whom any given emotion regulation strategy is effective in the immediate term (i.e., whether a strategy reduces negative affect immediately after being used; Aldao, 2013; Webb et al., 2012). Researchers have demonstrated that a multitude of factors can influence a given strategy's effectiveness (e.g., situational goals; English et al., 2017; perceived controllability of a stressor; Haines et al., 2016; timing of strategy use; Kalokerinos et al., 2017), which may call into question whether strategies can be reliably distinguished as adaptive or maladaptive. However, researchers studying BPD, and psychopathology more broadly, have consistently found empirical evidence that putatively adaptive and putatively maladaptive strategies can be differentiated based on individual differences (e.g., Aldao \& Nolen-Hoeksema, 2012; Naragon-Gainey et al., 2017; Neacsiu et al., 2010; Southward \& Cheavens, 2020) and within-person patterns of use (e.g., McMahon \& Naragon-Gainey, 2019). People with BPD in particular have demonstrated the ability to use strategies that are both effective at reducing negative emotions in the short-term and maladaptive in the long-term (e.g., self-harm; Carpenter \& Trull, 2013; Putnam \& Silk, 2005). Given that BPD is defined in part by a pervasive pattern of maladaptive behaviors present in a variety of contexts, we believe the adaptive-maladaptive distinction is a useful and appropriate general framework that can be enriched by more specific analyses.

Researchers have begun to apply EMA methods to understand how people with BPD and elevated BPD features behaviorally respond to negative emotions. When assessed five times per day for seven days, participants with elevated BPD features engaged in more frequent rumination 
in response to interpersonal stressors than those lower in BPD features (Yaroslavsky et al., 2019). In two separate studies, participants with BPD or elevated BPD features were assessed eight times per day for four to six days (Chapman et al., 2009; 2017). When instructed to use avoidance-oriented behaviors (e.g., pushing feelings away, suppressing facial expressions of emotions), participants reported decreases in momentary negative affect across independent studies. In contrast, when instructed to use approach-oriented behaviors (e.g., accepting negative emotions and thoughts), people with BPD or elevated BPD features reported increases in negative affect. These findings suggest that people with BPD or elevated BPD features may engage in more frequent putatively maladaptive behaviors in part because they are more effective at regulating their emotions in the moment. However, because participants were instructed to use approach- and avoidance-oriented strategies in Chapman et al. (2009) and Chapman et al. (2017), it is unclear if people with BPD would respond similarly without such direct prompting.

One of the difficulties in designing EMA studies is determining how frequently to sample participants' experiences. Given that people in the general population report experiencing emotions $90 \%$ of their waking hours (Trampe et al., 2015), more intensive sampling may be necessary to accurately capture the variety of emotional experiences in BPD. Across two studies, Ebner-Priemer et al. (2015) assessed participants with BPD every 15 min during waking hours for 24 hours or, in a third study, every 60 min during waking hours for four days. Across studies, people with BPD reported more intense negative affect and greater affective instability than healthy controls. Although people with BPD reported greater persistence of anxiety and sadness than healthy controls, neither the duration of nor instability in these emotions differed from other clinical controls (i.e., those with posttraumatic stress disorder or bulimia nervosa; Kockler et al., 
2017; Santangelo et al., 2014). People with BPD did, however, report more frequent experiences of anger than healthy and clinical controls (Kockler et al., 2019). Although anger was uniquely associated with greater distress beyond general emotional intensity for those with BPD, joy was uniquely associated with less distress beyond general emotional intensity.

These studies are valuable because they allow for direct comparisons of emotional responses between people with BPD, healthy controls, and clinical controls. However, in each of these studies, the EMA data collected was summarized in a single variable for each participant to be used in the group comparisons. Although appropriate for the questions of interest, the moment-to-moment relations among emotion variables is not captured in these analytic methods. As many others have previously noted, research questions regarding within-person effects must be studied at the within-person level (e.g., Fisher et al., 2018). Given that affective instability is inherently a longitudinal, within-person process, it is necessary to analyze the relations among emotions at the within-person level. Further, the fact that BPD is relatively rare (Torgersen, 2009) and yet extremely costly (Hastrup et al., 2019) indicates that studies that test within-person processes among those with BPD are valuable to enhance our understanding of the maintenance of the disorder. Finally, because all three studies 2 above relied on the same sample of 43 women from Germany with BPD indicates that independent samples of women and men with BPD are necessary to test the replicability and generalizability of these within-person processes.

\section{Current Study}

In the current study, we conducted a preliminary replication and extension of this work by examining variability in and relations between emotions and emotion regulation behaviors on up to 24 hourly occasions across two days in a sample of eight participants with BPD. This is a

2 Although we note that Ebner-Priemer et al. (2015) also included two independent datasets of 50 women with BPD from the US and 42 women with BPD from Germany. 
secondary data analysis from Sauer-Zavala et al. (2020). We hypothesized that people with BPD would report generally negative emotion intensities with high variability and instability. We further expected that participants would report frequent experiences of anger, sadness, and guilt/shame, consistent with the DSM-5 criteria for BPD. Given the centrality of emotion dysregulation to BPD, we hypothesized that participants would use putatively maladaptive strategies relatively more often than putatively adaptive strategies and that the use of maladaptive strategies would be associated with more negative emotion intensity at any given moment. Finally, we hypothesized that the use of maladaptive strategies would predict increases in positive emotion intensity from one hour to the next, whereas adaptive strategies would predict increases in negative emotion intensity, as previous research has shown that avoidanceoriented, maladaptive behaviors predicted increases in positive emotion intensity, whereas approach-oriented, adaptive behaviors predicted decreases in positive emotion intensity (Chapman et al., 2009; 2017).

\section{Materials \& Methods}

\section{Participants}

Eight individuals with BPD $\left(M_{\text {age }}=21.57, S D_{\text {age }}=3.05 ; 63 \%\right.$ female; $63 \%$ Asian; $88 \%$ non-Hispanic; 88\% not taking any psychotropic medications) participated in the current study. Inclusion criteria comprised the following: (1) a Diagnostic and Statistical Manual, 5th edition (DSM-5; APA, 2013) diagnosis of BPD, (2) willingness to maintain a stable dose of prescribed psychotropic medication and to refrain from obtaining additional psychosocial treatment for the duration of the study; (3) fluency in English; and (4) access to a personal smartphone. Participants were excluded if they (1) endorsed conditions that would require immediate treatment or hospitalization (e.g., current mania, schizophrenia or schizoaffective disorder, or 
organic mental disorder); (2) clear and acute suicidal intent; and (3) current or recent (within three months) drug dependence.

\section{Procedure}

Participants were recruited via online postings, requests to nearby treatment centers, and direct recruitment emails to those with BPD who had taken part in other studies conducted by the research team. Potential participants completed a brief phone screening; those deemed likely to be eligible were then invited to the laboratory where they provided informed consent and completed baseline self-report measures and in-person semi-structured assessments. Eligible participants were then instructed in the longitudinal study procedures.

The primary study from which these data are drawn, Sauer-Zavala et al. (2020), utilized a single-case experimental design (SCED; Barlow et al., 2009). Participants completed three study phases: a baseline phase (lasting two or four weeks), an intervention phase (lasting four weeks), and a follow-up phase (lasting four weeks). During the intervention phase, participants attended four weekly in-person sessions in which they learned and practiced the Countering Emotional Behaviors skill drawn from the Unified Protocol (UP). This skill involves (a) identifying the behavioral action urge associated with an experienced emotion and (b) acting in a way that is opposed to that urge. A full description of how this skill was taught is provided in Sauer-Zavala et al. (2020). Participants completed hourly EMA reports during two randomly chosen 12-hour blocks of time: one during the baseline phase and one during the last week of the treatment phase. These days were chosen to reduce overall participant burden in the context of other study procedures and to capture a snapshot of participants' functioning before and after the intervention phase. Participants received hourly notifications on their smartphones (12 total notifications over the 12-hour blocks of time) to respond to questions related to current 
emotional experiences, their antecedents, and subsequent behavioral responses. All study procedures were approved by the local university Institutional Review Board.

\section{Measures}

\section{Diagnostic Assessments}

\section{Structured Clinical Interview for DSM-IV Axis II Personality Disorders -}

Borderline Personality Disorder Module. The Structured Clinical Interview for DSM-IV Axis II Personality Disorders - Borderline Personality Disorder module (SCID-II-BPD; First et al., 1997) is a semi-structured diagnostic interview used to assess the presence or absence of BPD using DSM-5 criteria. The SCID-II-BPD has demonstrated excellent interrater reliability in previous research $(\kappa=.91$; Lobbestael et al., 2011) and in the current study $(\kappa=1.00$; SauerZavala et al., 2020). The SCID-II-BPD was administered by advanced doctoral students at participants' initial study visit.

Anxiety and Related Disorders Interview Schedule for DSM-5. The Anxiety and Related Disorders Interview Schedule for DSM-5 (ADIS-5; Brown \& Barlow, 2014) is a semistructured diagnostic interview used to assess the presence or absence of anxiety and related disorders using DSM-5 criteria. The ADIS has demonstrated excellent interrater reliability in previous research (Brown et al., 2001). In the current study, only modules from the ADIS-5 relevant to study exclusion criteria were administered (e.g., mania, psychosis, substance dependence, suicidality).

\section{Self-Reported Measures}

Demographics. Participants reported demographic characteristics at baseline in the laboratory. These characteristics included age, gender, racial and ethnic background, and current psychotropic medications (if any). 
Emotions and Emotional Intensity. Participants were asked to rate the intensity of their current emotional experience from 0 (no intensity at all) to 100 (greatest possible intensity). If participants answered with a score of " 0 ", the survey would end for that time point. After identifying the intensity of their emotional experience, participants were asked to select which of the following emotions best described how they were currently feeling: anger, sadness, anxiety, guilt/shame, or joy/happiness. These emotions were chosen because the negatively-valenced emotions provide relatively good coverage of the affective circumplex (Russell, 1980) and because these emotions are frequently targeted in evidence-based treatments for BPD (e.g., Dialectical Behavior Therapy [DBT]; Linehan, 1993).

Behavioral Responses. Once a specific emotion was selected, participants were asked to select one of five options that best described what they were doing in response to the emotion. These options were chosen to provide relatively broad (within the limitations of requesting hourly EMA responses) conceptual coverage of the range of responses people with BPD may use to regulate their emotions. Thus, these responses covered (1) problem-focused, putatively adaptive, behavioral regulation (i.e., problem-solving); (2) emotion-focused, putatively adaptive, cognitive regulation (i.e., acceptance); (3) emotion-focused, putatively maladaptive, behavioral regulation (i.e., engaging in impulsive behavior); (4) emotion-focused, putatively maladaptive, cognitive regulation (i.e., pushing the emotion away); and (5) emotion-focused, cognitive and behavioral regulation (i.e., digging into, or savoring, the emotion) that may be adaptive in response to positively-valenced emotions (e.g., joy/happiness) and maladaptive in response to negatively-valenced emotions (e.g., anger, anxiety, guilt/shame, sadness). Each option was accompanied by idiosyncratic descriptions (in plain English) of example behaviors unique to the endorsed emotion. For example, if participants endorsed anger, digging into the emotion was 
described as "digging in to the feeling (e.g., listening to angry music, venting, pacing)"; if they endorsed "sadness", digging into the emotion was described as "digging into the feeling (e.g., isolating myself, crying, listening to sad music, watching a sad movie)". A full list of the plain English descriptions presented is provided in Table S1 (Supplemental Online Materials).

\section{Analytic Method}

Because the five emotions assessed differ in valence (Russell, 1980) but were assessed only on the dimension of intensity, we multiplied participants' intensity ratings by -1 if they experienced an emotion other than joy/happiness in line with previous research practices (EbnerPriemer et al., 2015). Intensity ratings could thus range from -100-100.

Because participants were assessed on separate days before and after the behavioral intervention, we first examined whether participants' emotional responses differed between days. Given the small sample size, we conducted Wilcoxon signed-rank tests to examine these differences, which provide a non-parametric alternative to paired-sample $t$-tests more appropriate for small samples because they do not assume variables are normally distributed. For the same reasons, we also calculated Spearman's rank correlation coefficients to compare participants' emotional responses on separate days. We compared emotion intensity, using measures of the mean, variability (i.e., within-person standard deviations), and instability (i.e., mean of squared successive differences [MSSD; Jahng et al., 2008]), emotion frequency, and emotion regulation frequency between days 1 and 2 using the wilcox.test and cor.test (with the Spearman specifier) functions in R (Version 3.4.1; R Core Team, 2017).

Next, we assessed descriptive statistics characterizing the frequency of participants' specific emotions and emotion regulation behaviors. Using hierarchical linear modeling (HLM) with proc mixed in SAS Version 9.4 to account for the multilevel structure of the data, we 
explored whether emotion intensity was related to specific emotions, over and above the day and time of day assessed. We used a compound symmetry residual covariance structure to (a) model repeated observations nested within days and participants and (b) model repeated observations on different days to account for potential differences between days. Finally, we tested (a) whether specific regulation behaviors were associated with emotion intensity, in separate models, and (b) whether specific regulation behaviors predicted changes in emotion intensity from one hour to the next, in separate models, using the same HLM model specifications above. Although there is as yet no consensus on the sample sizes needed to conduct reliable HLMs, simulation work has suggested that analyses conducted with ten people assessed five times provide relatively unbiased estimates of level-1 (i.e., momentary) predictors (Maas \& Hox, 2005). The authors of this study note that level-2 (i.e., person-level) predictors in designs with these characteristics are more substantially biased. However, given that our questions of interest concern level-1 relations, we proceeded with our analytic plan. All code and supplemental materials can be found at https://doi.org/10.17605/OSF.IO/CUA5M.

\section{Results}

\section{Differences Between Days}

Participants provided a total of 150 responses. The average emotion intensity did not significantly differ between day $1(M=-14.78, S D=15.90)$ and day $2(M=-15.34, S D=$ 41.18), $V=17, p=.95,95 \% \mathrm{CI}[-36.65,37.23], d=.02$. This lack of difference is borne out when visually inspecting the idiographic patterns of participants' emotion intensities at each timepoint (Figure 1). Dimensionally, emotion intensity from day 1 to day 2 exhibited a nonsignificant but medium-sized positive association, $\rho=.33, p=.43$. Averaging across days, participants tended to report more negative emotion intensity $(M=-15.06, S D=30.15)$ that, 
however, was not significantly different from $0, V=6, p=.11,95 \%$ CI $[-36.50,11.80]$. Withinperson standard deviations of emotion intensity also did not differ between day $1(M=38.85, S D$ $=26.79)$ and day $2(M=51.95, S D=14.95), V=7, p=.30,95 \% \mathrm{CI}[-41.59,11.70], d=.60$. Averaging across days, participants reported a relatively large average standard deviation $(M=$ 45.40, $S D=21.92)$. Similarly, emotion intensity MSSD did not differ between day $1(M=$ $4159.54, S D=4635.77)$ and day $2(M=5024.76, S D=3577.02), V=13, p=.94,95 \% \mathrm{CI}[-$ 6599.40, 4941.88], $d=.20$. Dimensionally, emotion intensity MSSD from day 1 was unrelated to MSSD from day $2, \rho=.04, p=.96$.

The frequency with which participants reported specific emotions did not differ from day 1 to day $2, p \mathrm{~s}>.12$ (Table $\mathrm{S} 2$ ). The frequency with which participants reported using pushing away and digging in did not differ from day 1 to day 2, ps > .06 (Table S3). However, participants reported greater average frequency of using problem-solving on day $1(M=2.88, S D$ $=1.73)$ than day $2(M=1.38, S D=1.19), V=28, p=.02, d=1.01$, and greater average frequency of pushing emotions away on day $1(M=3.17, S D=1.83)$ than day $2(M=1.17, S D=$ 1.47), $V=21, p=.03, d=.72$.

\section{Frequency of Emotions and Emotion Regulation Behaviors}

Participants reported experiencing anxiety most frequently $(n=67,44.7 \%$; Table 1$)$, followed by joy $(n=52,34.7 \%)$. Participants reported experiencing all other emotions less than $10 \%$ of the time. Of note, participants reported experiencing each of these emotions at similar levels of intensity, $F(4,122)=.58, p=.68$. Participants reported most frequently digging into their emotional experiences $(n=54,38.0 \%$; Table 2$)$, followed by problem-solving $(n=34$, $23.9 \%)$, pushing away $(n=26,18.3 \%)$, and acceptance $(n=24,16.9 \%)$. Participants reported impulsive behaviors relatively rarely $(n=4,2.8 \%)$. There was a significant difference in which 
emotion regulation behaviors were used in response to specific emotions, $\chi_{2}(16)=91.45, p<$ .01. Specifically, digging into emotional experiences was used more frequently in response to joy $(n=44,31.0 \%$; Table 3$)$ than other emotions. Problem-solving $(n=26,18.3 \%)$, pushing emotions away $(n=17,12.0 \%)$, and acceptance $(n=14,9.9 \%)$ were each used more frequently in response to anxiety than other emotions. Impulsive behaviors were used relatively rarely and only in response to joy and anxiety.

\section{Temporal Relations Among Emotion Regulation Behaviors and Emotion Intensity}

Finally, we examined which emotion regulation behaviors were associated with emotion intensity at the same time point and which behaviors predicted changes in emotion intensity from one hour to the next. Digging into the emotional experience was associated with a more positive emotion intensity, $B=68.63, S E=7.67, p<.01,95 \%$ CI $[53.45,83.81]$ (Table S4). On the other hand, both pushing emotions away, $B=-41.93, S E=10.41, p<.01,95 \%$ CI $[-62.54,-21.32]$ (Table S5) and problem-solving, $B=-25.08, S E=9.84, p=.01,95 \%$ CI $[-44.55,-5.61]$ (Table S6), were associated with more negative emotion intensities. Neither acceptance, $B=-21.27, S E$ $=11.69, p=.07,95 \% \mathrm{CI}[-44.41,1.88]$ (Table S7), nor impulsive behaviors, $B=12.92, S E=$ 25.03, $p=.61,95 \%$ CI $[-36.62,62.47]$ (Table S8), were significantly associated with emotion intensities.

Only acceptance and pushing emotions away predicted hour-to-hour changes in emotion intensity. Acceptance predicted increasing negative emotion intensities from one hour to the next, $B=-30.19, S E=13.23, p=.02,95 \%$ CI $[-56.43,-3.95]$ (Table S9). Pushing emotions away predicted increasing positive emotion intensities from one hour to the next, $B=27.92, S E=$ $12.42, p=.03,95 \%$ CI $[3.30,52.53]$ (Table S10). All other emotion regulation behaviors were unrelated to changes in emotion intensity (Tables S11-S13). 


\section{Discussion}

In this preliminary intensive EMA study of participants with BPD, we found that people with BPD tend to report neutral-to-negative emotional experiences throughout a given day that is relatively variable both within a day and from one day to the next. This finding replicates previous EMA work comparing the valence, variability, and instability of affect experienced by those with BPD with others (Berenson et al., 2011; Ebner-Priemer et al., 2007, 2015; Glaser et al., 2008; Houben et al., 2018; Stiglmayr et al., 2005; Trull et al., 2008; Wolff et al., 2007) and extends these results to an absolute metric of affective valence measured hourly throughout individual days.

When discrete emotional responses were assessed, participants in the current study most frequently reported experiences of anxiety and joy. These results were consistent with a sample of American undergraduate students oversampled to be elevated in neuroticism (Heiy \& Cheavens, 2014; Southward et al., 2019). However, these results differed somewhat from a German sample of people with BPD (Kockler et al., 2019) in which participants reported experiencing anger most frequently, followed closely by sadness and anxiety. These differences may result from sampling variability. For instance, participants in the current study were excluded for current imminent suicidality, whereas this was not an explicit exclusion criterion in Kockler et al. (2019). This difference in exclusion criteria may have resulted in a less clinically severe sample of BPD participants in the current study that was more similar to undergraduates with elevated neuroticism. Alternatively, by measuring discrete emotions with single-item indicators, which tend to be less reliable than dimensional measures of affect (Barrett, 1998; 2006), the differences in study results may have been artificially exaggerated. It is thus possible that intensity of negative affect could be similar among participants in both studies even if the 
specific emotions reported differed. Finally, these differences may have resulted from normal sample variability, given the relatively small samples used in both studies, or cultural differences in the experience of BPD between the US and Germany.

In terms of behavioral responses, participants with BPD in the current study reported most frequently digging into emotions and this strategy was used most often in response to joy/happiness, leading to a positive association between digging in and positive emotion intensity. Digging into the emotion of joy/happiness is conceptually similar to the strategy of savoring emotions (e.g., "I tried to revel in the moment and concentrate on how good I felt"; Heiy \& Cheavens, 2014). Savoring positive emotions was also the most frequently-endorsed strategy used by undergraduates oversampled for neuroticism (Heiy \& Cheavens, 2014). This may suggest that people with BPD are engaging in adaptive behavioral responses to positive emotions. However, people with BPD may experience more instances of negative affect that reduce the perceived capacity or effectiveness of adaptive strategies. Participants in the current study used problem-solving more often than acceptance or pushing emotions away, whereas undergraduates oversampled for neuroticism used problem-solving less frequently than these two strategies (Heiy \& Cheavens, 2014). This pattern of behaviors may indicate (1) that people with BPD are adaptively problem-solving difficult situations relatively more often, (2) that they are faced with more frequent stressful situations, or (3) that problem-solving is being used as a form of distraction. Future researchers are encouraged to include healthy and clinical control groups to both test the functional nature of these hypotheses to determine which patterns of behaviors are relative strengths for those with BPD compared to others.

Surprisingly, all emotions assessed were experienced at similar levels of intensity, on average, across participants. Because anxiety was experienced most frequently by participants in 
the current study, these results suggest that, over the course of a given day, people with BPD may most often experience anxiety while other emotions are experienced at a similar level of intensity. It is important to note that emotional intensity is only one dimension of the impact of emotions on a person's life. Emotional intensity may not indicate how functionally impairing an emotion is, and we encourage future researchers to directly assess this domain. Further, by averaging across emotional experiences, these analyses may be obscuring moments in which particularly intense or interfering emotions arose. To more accurately capture these dynamics, we encourage future researchers to use event-contingent reporting so participants can more directly connect the experience of particularly intense emotions with their consequences. We believe this would be feasible given previous work demonstrating similar levels of data quality and number of responses regarding affective and behavioral outcomes between randomly scheduled and event-contingent reporting (Himmelstein et al., 2019).

We found mixed support for our hypothesis regarding the relation between adaptive and maladaptive strategies and momentary emotional intensity. Both pushing away, a putatively maladaptive strategy, and problem-solving, a putatively adaptive strategy, were associated with greater negative emotion intensity. These results may indicate two contrasting ways people with BPD attempt to manage anxiety. That is, for some people with BPD, more intense anxiety may prompt them to try to push the emotion away, while for others, it may be a signal to problemsolve around the source of the anxiety. Because pushing emotions away predicted an increase in positive emotion intensity, in line with our hypotheses and previous research (Chapman et al., 2009; 2017), these results suggest that pushing emotions away is an effective, short-term strategy that people with BPD use to manage more intense negative emotions and especially anxiety. Problem-solving, in contrast, did not predict increases in positive emotion intensity, in contrast to 
our hypothesis, which may indicate why it is less immediately reinforcing for people with BPD to use. Alternatively, this finding may suggest that problem-solving predicts immediate functional outcomes (e.g., resolving an argument with a close other), rather than emotional outcomes. We encourage future researchers to include measures of functional outcomes to test the broader effects of the emotion regulation strategies in participants' repertoires.

In line with our hypotheses, acceptance, a putatively adaptive strategy, predicted hour-byhour increases in negative emotion intensity, and pushing emotions away, a putatively maladaptive strategy, predicted hour-by-hour increases in positive emotion intensity. Further, pushing emotions away was more likely to be used in response to more intense negative emotions, also in line with our hypotheses. These results are consistent with previous EMA studies of BPD in which instructed acceptance was associated with increases in negative emotions, whereas instructed pushing away of emotions was associated with decreases in negative emotions (Chapman et al., 2009; 2017). Because acceptance is designed to bring awareness to one's personal experience as it is, whereas pushing emotions away is designed to ignore one's personal experience, and because people with BPD tend to experience more frequent and intense negative emotions, it is less surprising that acceptance would enhance these negative emotions and pushing emotions away would reduce them. These results may also highlight the difficulty of using certain putatively adaptive strategies by people with BPD, as these strategies may not be viewed as effective or reinforcing by the person using them. Similarly, these results may highlight the difficulty of reducing the use of more maladaptive strategies by those with BPD because they are more effective and reinforcing in the moment. Alternatively, acceptance may be an underdeveloped skill for people with BPD (Southward \& Cheavens, 2020), who may have instead practiced resignation or rumination instead of 
nonjudgmental, present-moment acceptance. In contrast, undergraduate students oversampled for neuroticism reported that acceptance was associated with better and improving moods, whereas emotional suppression, which is conceptually similar to pushing emotions away, was unrelated to changes in mood (Heiy \& Cheavens, 2014; Southward et al., 2019). Together, these results may suggest that acceptance is perceived as more effective in the moment if it can be used in response to more positively-valenced emotions, whereas suppression is viewed as more effective if it can be used in response to more negatively-valenced emotions. Researchers interested in improving treatments for BPD are encouraged to investigate how to navigate these short-term difficulties of using acceptance and reducing pushing emotions away to determine how, when, and for whom to attempt to alter patients' emotion regulation repertoires.

The results of this study should be considered in light of its limitations. Given that the data are drawn from a single-case experimental design, the sample size is relatively small, making it difficult to generalize our conclusions beyond people with BPD without imminent suicidal risk. Further, because a control group was not included in the study, we cannot determine whether these patterns of emotions and behaviors are unique to BPD or more transdiagnostic. Although we assessed participants frequently and statistically accounted for changes in emotions from one time point to the next, because this was an observational study, we cannot determine the direction of causal effects among variables if they exist. Similarly, because we did not assess for additional contextual factors (e.g., participants' regulatory goal[s] in a given situation; the perceived controllability of the stressor), we cannot determine the relative strength of associations between these factors with emotional outcomes. Because we used emotion intensity as our outcome of interest and did not assess participants' idiographic emotion regulation goals in each moment, our definition of emotion regulation effectiveness is limited to 
hedonic effectiveness. We encourage future basic and treatment researchers (especially those who study treatments that explicitly consider situational context such as DBT; Linehan, 1993) to incorporate these factors in their assessment batteries. Because we asked participants to identify the single most salient emotion and single most applicable behavior, the variability around our measures of interest was relatively limited. Finally, although we provided plain English descriptions of each behavior, it is possible that participants did not accurately report what they did or engaged in unlisted behaviors instead of or in addition to the options presented to them (Opitz et al., 2015). Future researchers are encouraged to extend these results by recruiting larger samples and assessing a wider variety of emotions and theoretically important behaviors.

Despite these limitations, we were able to collect intensive measurements from a difficult-to-reach population regarding not only their experience of emotions but how they behaviorally responded to them. This data-driven method can help clarify the daily lived experience of people with BPD to more accurately characterize their experiences. These results can further inform our understanding of the relative strengths of people with BPD and demonstrate novel associations for clinical assessment and treatment researchers to replicate and potentially intervene on. 


\section{References}

Aldao, A. (2013). The future of emotion regulation research: Capturing context. Perspectives on Psychological Science, 8(2), 155-172. https://doi.org/10.1177/1745691612459518

Aldao, S., \& Nolen-Hoeksema, S. (2012). When are adaptive strategies most predictive of psychopathology? Journal of Abnormal Psychology, 121(1), 276-281. https://doi.org/10.1037/a0023598

Aldao, A., Nolen-Hoeksema, S., \& Schweizer, S. (2010). Emotion-regulation strategies across psychopathology: A meta-analytic review. Clinical Psychology Review, 30(2), 217-237. https://doi.org/10.1016/j.cpr.2009.11.004

American Psychiatric Association. (2013). Personality disorders. In Diagnostic and statistical manual of mental disorders (5th ed.). Author. https://doi.org/10.1176/appi.books.9780890425596.dsm18

Barlow, D. H., Nock, M. K., \& Hersen, M. (2009). Single case experimental designs: Strategies for studying behavior change (3rd ed.). Pearson.

Barrett, L. F. (1998). Discrete emotions or dimensions? The role of valence focus and arousal focus. Cognition and Emotion, 12(4), 579-599. https://doi.org/10.1080/026999398379574

Barrett, L. F. (2006). Solving the emotion paradox: Categorization and the experience of emotion. Personality and Social Psychology Review, 10(1), 20-46. https://doi.org/10.1207/s15327957pspr1001_2

Berenson K. R., Downey, G., Rafaeli, E., Coifman, K. G., \& Paquin, N. L. (2011). The rejectionrage contingency in borderline personality disorder. Journal of Abnormal Psychology, 120(3), 681-690. https://doi.org/10.1037/a0023335 
Brown, T. A., \& Barlow, D. H. (2014). Anxiety and related disorders interview schedule for DSM-5 (ADIS-5) - adult version. Oxford University Press.

Brown, T. A., Di Nardo, P. A., Lehman, C. L., \& Campbell, L. A. (2001). Reliability of DSM-IV anxiety and mood disorders: Implications for the classification of emotional disorders. Journal of Abnormal Psychology, 110(1), 49-58. https://doi.org/10.1037//0021-843x.110.1.49

Carpenter, R. W., \& Trull, T. J. (2013). Components of emotion dysregulation in borderline personality disorder: A review. Current Psychiatry Reports, 15, 335. https://doi.org/10.1007/s11920-012-0335-2

Chapman, A. L. (2019). Borderline personality disorder and emotion dysregulation. Development and Psychopathology, 31, 1143-1156. https://doi.org/10.1017/S0954579419000658

Chapman, A. L., Rosenthal, M. Z., Dixon-Gordon, K. L., Turner, B. J., \& Kuppens, P. (2017). Borderline personality disorder and the effects of instructed emotional avoidance or acceptance in daily life. Journal of Personality Disorders, 31(4), 483-502. https://doi.org/10.1521/pedi_2016_30_264

Chapman, A. L., Rosenthal, M. Z., \& Leung, D. W. (2009). Emotion suppression in borderline personality disorder: An experience sampling study. Journal of Personality Disorders, 23(1), 29-47. https://doi.org/10.1521/pedi.2009.23.1.29

Cheavens, J. S., \& Heiy, J. E. (2011). The differential roles of affect and avoidance in major depressive and borderline personality disorder symptoms. Journal of Social and Clinical Psychology, 30(5), 441-457. https://doi.org/10.1521/jscp.2011.30.5.441 
Cowdry, R. W., Gardner, D. L., O’Leary, K. M., Leibenluft, E., \& Rubinow, D. R. (1991). Mood variability: A study of four groups. American Journal of Psychiatry, 148(11), 1505-1511. https://doi.org/10.1176/ajp.148.11.1505

Crowell, S. E., Beauchaine, T. P., \& Linehan, M. M. (2009). A biosocial developmental model of borderline personality: Elucidating and extending Linehan's theory. Psychological Bulletin, 135(3), 495-510. https://doi.org/10.1037/a0015616

Dick, A. M., \& Suvak, M. K. (2018). Borderline personality disorder affective instability: What you know impacts how you feel. Personality Disorders: Theory, Research, and Treatment, 9(4), 369-378. https://doi.org/10.1037/per0000280

Ebner-Priemer, U. W., Houben, M., Santangelo, P., Kleindienst, N., Tuerlinckx, F., Oravecz, Z., Verleysen, G., Van Deun, K., Bohus, M., \& Kuppens, P. (2015). Unraveling affective dysregulation in borderline personality disorder: A theoretical model and empirical evidence. Journal of Abnormal Psychology, 124(1), 186-198. https://doi.org/10.1037/abn0000021

Ebner-Priemer, U. W., Kuo, J., Kleindienst, N., Welch, S. S., Reisch, T., Reinhard, I., Lieb, K., Linehan, M. M., \& Bohus, M. (2007). State affective instability in borderline personality disorder assessed by ambulatory monitoring. Psychological Medicine, 37(7), 961-970. https://doi.org/10.1017/S0033291706009706

Ebner-Priemer, U. W., Welch, S. S., Grossman, P., Reisch, T., Linehan, M. M., \& Bohus, M. (2007). Psychophysiological ambulatory assessment of affective dysregulation in borderline personality disorder. Psychiatry Research, 150(3), 265-275. https://doi.org/10.1016/j.psychres.2006.04.014 
English, T., Lee, I. A., John, O. P., \& Gross, J. J. (2017). Emotion regulation strategy selection in daily life: The role of social context and goals. Motivation and Emotion, 41, 230-242. https://doi.org/10.1007/s11031-016-9597-z

First, M. B., Spitzer, R. L., Gibbon, M., Williams, J. B. W., \& Benjamin, L. S. (1997). Structured clinical interview for DSM-IV axis II personality disorders (SCID-II). American Psychiatric Press.

Fisher, A. J., Medaglia, J. D., \& Jeronimus, B. F. (2018). Lack of group-to-individual generalizability is a threat to human subjects research. PNAS, 115(27), E6106-E6115. https://doi.org/10.1073/pnas.1711978115

Glaser, J.-P., Os, J. V., Mengelers, R., \& Myin-Germeys, I. (2008). A momentary assessment study of the reputed emotional phenotype associated with borderline personality disorder. Psychological Medicine, 38(9), 1231-1239. https://doi.org/10.1017/S0033291707002322

Grant, B. F., Chou, S. P., Goldstein, R. B., Huang, B., Stinson, F. S., Saha, T. D., Smith, S. M., Dawson, D. A., Pulay, A. J., Pickering, R. P., \& Ruan, W. J. Prevalence, correlates, disability, and comorbidity of DSM-IV borderline personality disorder: Results from the Wave 2 National Epidemiologic Survey on Alcohol and Related Conditions. Journal of Clinical Psychiatry, 69(4), 533-545. https://doi.org/10.4088/jcp.v69n0404

Gunderson, J. G., \& Links, P. S. (2008). Borderline personality disorder: A clinical guide (2nd ed.). American Psychiatric Publishing, Inc.

Haines, S. J., Gleeson, J., Kuppens, P., Hollenstein, T., Ciarrochi, J., Labuschagne, I., Grace, C., \& Koval, P. (2016). The wisdom to know the difference: Strategy-situation fit in emotion regulation in daily life is associated with well-being. Psychological Science, 27(12), 1651-1659. https://doi.org/10.1177/0956797616669086 
Hastrup, L. H., Jennum, P., Ibsen, R., Kjellberg, J., \& Simonsen, E. (2019). Societal costs of borderline personality disordes: A matched-controlled nationwide study of patients and spouses. Acta Psychiatrica Scandinavica, 140(5), 458-467. https://doi.org/10.1111/acps.13094

Heiy, J. E., \& Cheavens, J. S. (2014). Back to basics: A naturalistic assessment of the experience and regulation of emotion. Emotion, 14(5), 878-891. https://doi.org/10.1037/a0037231

Himmelstein, P. H., Woods, W. C., \& Wright, A. (2019). A comparison of signal- and eventcontingent ambulatory assessment of interpersonal behavior and affect in social situations. Psychological Assessment, 31(7), 952-960. https://doi.org/10.1037/pas0000718

Houben, M., Claes, L., Sleuwaegen, E., Berens, A., \& Vansteelandt, K. (2018). Emotional reactivity to appraisals in patients with borderline personality disorder: A daily life study. Borderline Personality Disorder and Emotion Dysregulation, 5, 18. https://doi.org/10.1186/s40479-018-0095-7

Jahng, S., Wood, P. K., \& Trull, T. J. (2008). Analysis of affective instability in ecological momentary assessment: Indices using successive difference and group comparison via multilevel modeling. Psychological Methods, 13(4), 354-375. https://doi.org/10.1037/a0014173

Kalokerinos, E. K., Résibois, M., Verduyn, P., \& Kuppens, P. (2017). The temporal deployment of emotion regulation strategies during negative emotional episodes. Emotion, 17(3), 450-458. https://doi.org/10.1037/emo0000248

Kockler, T. D., Santangelo, P. S., Limberger, M. F., Bohus, M., \& Ebner-Priemer, U. W. (2019). Specific or transdiagnostic? The occurrence of emotions and their association with 
distress in the daily life of patients with borderline personality disorder compared to clinical and healthy controls. Psychiatry Research, 284, 112692. https://doi.org/10.1016/j.psychres.2019.112692

Kockler, T. D., Tschacher, W., Santangelo, P. S., Limberger, M. F., \& Ebner-Priemer, U. W. (2017). Specificity of emotion sequences in borderline personality disorder compared to posttraumatic stress disorder, bulimia nervosa, and healthy controls: An e-diary study. Borderline Personality Disorder and Emotion Dysregulation, 4, 26. https://doi.org/10.1186/s40479-017-0077-1

Kring, A. M., \& Sloan, D. M. (2010). Emotion regulation and psychopathology: A transdiagnostic approach to etiology and treatment. Guilford Press.

Kuo, J. R., Neacsiu, A. D., Fitzpatrick, S., \& MacDonald, D. E. (2014). A methodological examination of emotion inductions in borderline personality disorder: A comparison of standardized versus idiographic stimuli. Journal of Psychopathology and Behavioral Assessment, 36(1), 155-164. https://doi.org/10.1007/s10862-013-9378-x

Linehan, M. M. (1993). Cognitive-behavioral treatment of borderline personality disorder. Guilford Press.

Lobbestael, J., Leurgans, M., \& Arntz, A. (2011). Inter-rater reliability of the Structured Clinical Interview for DSM-IV Axis I Disorders (SCID I) and Axis II Disorders (SCID II). Clinical Psychology and Psychotherapy, 18(1), 75-79. https://doi.org/10.1002/cpp.693

Maas, C. J., \& Hox, J. J. (2005). Sufficient sample sizes for multilevel modeling. Methodology, 1(3), 86-92. https://doi.org/10.1027/1614-1881.1.3.86

McMahon, T. P., \& Naragon-Gainey, K. (2019). The multilevel structure of daily emotionregulation-strategy use: An examination of within- and between-person associations in 
naturalistic settings. Clinical Psychological Science, 7(2), 321-339.

https://doi.org/10.1177/2167702618807408

Naragon-Gainey, K., McMahon, T. P., \& Chacko, T. P. (2017). The structure of common emotion regulation strategies: A meta-analytic examination. Psychological Bulletin, 143(4), 384-427. https://doi.org/10.1037/bul0000093

Neacsiu, A. D., Bohus, M., \& Linehan, M. M. (2013). Dialectical behavior therapy skills: An intervention for emotion dysregulation. In J. J. Gross (Ed.), Vol. 2. Handbook of emotion regulation (pp. 491-508). Guilford Press.

Neacsiu, A. D., Rizvi, S. L., Vitaliano, P. P., Lynch, T. R., \& Linehan, M. M. (2010). The dialectical behavior therapy ways of coping checklist: Development and psychometric properties. Journal of Clinical Psychology, 66(6), 563-582.

https://doi.org/10.1002/jclp.20685

Nica, E. I., \& Links, P. S. (2009). Affective instability in borderline personality disorder: Experience sampling findings. Current Psychiatry Reports, 11(1), 74-81. https://doi.org/10.1007/s11920-009-0012-2

Opitz, P. C., Cavanagh, S. R., \& Urry, H. L. (2015). Uninstructed emotion regulation choice in four studies of cognitive reappraisal. Personality and Individual Differences, 86, 455464. https://doi.org/10.1016/j.paid.2015.06.048

Putnam, K. M., \& Silk, K. R. (2005). Emotion dysregulation and the development of borderline personality disorder. Development and Psychopathology, 17(4), 899-925. https://doi.org/10.1017/s0954579405050431

R Core Team. (2017). R: A language and environment for statistical computing. R Foundation for Statistical Computing. https://www.R-project.org/ 
Reich, D. B., Zanarini, M. C., Hopwood, C. J., Thomas, K. M., \& Fitzmaurice, G. M. (2014). Comparison of affective instability in borderline personality disorder and bipolar disorder using a self-report measure. Personality and Mental Health, 8(2), 143-150. https://doi.org/10.1002/pmh.1247

Richetin, J., Preti, E., Costantini, G., \& De Panfilis, C. (2017) The centrality of affective instability and identity in borderline personality disorder: Evidence from network analysis. PLoS ONE, 12(10), e0186695. https://doi.org/10.1371/journal.pone.0186695

Rosenthal, M. Z., Gratz, K. L., Kosson, D. S., Cheavens, J. S., Lejuez, C. W., \& Lynch, T. R. (2008). Borderline personality disorder and emotional responding: A review of the research literature. Clinical Psychology Review, 28(1), 75-91. https://doi.org/10.1016/j.cpr.2007.04.001

Russell, J. A. (1980). A circumplex model of affect. Journal of Personality and Social Psychology, 39(6), 1161-1178.https://doi.org/10.1037/h0077714

Santangelo, P., Bohus, M., \& Ebner-Priemer, U. W. (2014). Ecological momentary assessment in borderline personality disorder: A review of recent findings and methodological challenges. Journal of Personality Disorders, 28(4), 555-576. https://doi.org/10.1521/pedi_2012_26_067

Santangelo, P., Mussgay, L., Sawitzki, G., Trull, T. J., Reinhard, I., Steil, R., Klein, C., Bohus, M., \& Ebner-Priemer, U. W. (2014). Specificity of affective instability in patients with borderline personality disorder compared to posttraumatic stress disorder, bulimia nervosa, and healthy controls. Journal of Abnormal Psychology, 123(1), 258-272. https://doi.org/10.1037/a0035619 
Sauer-Zavala, S., Cassiello-Robbins, C., Woods, B. K., Curreri, A., Wilner Tirpak, J., \& Rassaby, M. (2020). Countering emotional behaviors in the treatment of borderline personality disorder. Personality Disorders: Theory, Research, \& Treatment. Advance online publication. https://doi.org/10.1037/per0000379

Southward, M. W., \& Cheavens, J. S. (2018). Identifying core deficits in a dimensional model of borderline personality disorder features: A network analysis. Clinical Psychological Science, 6(5), 685-703. https://doi.org/10.1177/2167702618769560

Southward, M. W., \& Cheavens, J. S. (2020). Quality or quantity? A multistudy analysis of emotion regulation skills deficits associated with borderline personality disorder. Personality Disorders: Theory, Research, and Treatment, 11(1), 24-35. https://doi.org/10.1037/per0000357

Southward, M. W., Heiy, J. E., \& Cheavens, J. S. (2019). Emotions as context: Do the naturalistic effects of emotion regulation strategies depend on the regulated emotion? Journal of Social and Clinical Psychology, 38(6), 451-474. https://doi.org/10.1521/jscp.2019.38.6.451

Stiglmayr, C. E., Grathwol, T., Linehan, M. M., Ihorst, G., Fahrenberg, J., \& Bohus, M. (2005). Aversive tension in patients with borderline personality disorder: A computer-based controlled field study. Acta Psychiatrica Scandinavica, 111(5), 372-379. https://doi.org/10.1111/j.1600-0447.2004.00466.x

Torgersen, S. (2009). The nature (and nurture) of personality disorders. Scandinavian Journal of Psychology, 50(6), 624-632. https://doi.org/10.1111/j.1467-9450.2009.00788.x 
Tragesser, S. L., \& Robinson, R. J. (2009). The role of affective instability and UPPS impulsivity in borderline personality disorder features. Journal of Personality Disorders, 23(4), 370383. https://doi.org/10.1521/pedi.2009.23.4.370

Trampe, D., Quoidbach, J. \& Taquet, M. (2015). Emotions in everyday life. PLoS ONE, 10(12), e0145450. https://doi.org/10.1371/journal.pone.0145450

Trull, T. J., Solhan, M. B., Tragesser, S. L., Jahng, S., Wood, P. K., Piasecki, T. M., \& Watson, D. (2008). Affective instability: Measuring a core feature of borderline personality disorder with ecological momentary assessment. Journal of Abnormal Psychology, 117(3), 647-661. https://doi.org/10.1037/a0012532

Webb, T. L., Miles, E., \& Sheeran, P. (2012). Dealing with feeling: A meta-analysis of the effectiveness of strategies derived from the process model of emotion regulation. Psychological Bulletin, 138(4), 775-808. https://doi.org/10.1037/a0027600

Widiger, T. A., \& Trull, T. J. (1993). Borderline and narcissistic personality disorders. In P. B. Sutker \& H. E. Adams (Eds.), Comprehensive handbook of psychopathology (p. 371394). Plenum Press. https://doi.org/10.1007/978-1-4615-3008-4_15

Wolff, S., Stiglmayr, C., Bretz, H. J., Lammers, C.-H., \& Auckenthaler, A. (2007). Emotion identification and tension in female patients with borderline personality disorder. British Journal of Clinical Psychology, 46(3), 347-360. https://doi.org/10.1348/014466507X173736

Yaroslavsky, I., Napolitano, S. C., \& France, C. M. (2019). Ruminative responses to interpersonal precipitants mediate borderline personality disorder features' effects on distress reactivity and recovery in daily life. Journal of Clinical Psychology, 75(12), 2188-2209. https://doi.org/10.1002/jclp.22839 


\section{Table 1}

Frequency of Specific Emotions

\begin{tabular}{cccccc} 
Participant & Anger & Sadness & Anxiety & Guilt/Shame & Joy \\
\hline 1 & $1(7.1 \%)$ & $2(14.3 \%)$ & $2(14.3 \%)$ & $1(7.1 \%)$ & $8(57.1 \%)$ \\
2 & $0(0 \%)$ & $0(0 \%)$ & $7(31.8 \%)$ & $0(0 \%)$ & $15(68.2 \%)$ \\
3 & $4(16.0 \%)$ & $2(8.0 \%)$ & $4(16.0 \%)$ & $0(0 \%)$ & $15(60.0 \%)$ \\
4 & $1(3.8 \%)$ & $2(7.7 \%)$ & $16(61.5 \%)$ & $2(7.7 \%)$ & $5(19.2 \%)$ \\
5 & $0(0 \%)$ & $2(100.0 \%)$ & $0(0 \%)$ & $0(0 \%)$ & $0(0 \%)$ \\
6 & $1(6.3 \%)$ & $2(12.5 \%)$ & $11(68.8 \%)$ & $0(0 \%)$ & $2(12.5 \%)$ \\
7 & $4(21.1 \%)$ & $0(0 \%)$ & $6(31.6 \%)$ & $0(0 \%)$ & $2(10.5 \%)$ \\
8 & $2(7.7 \%)$ & $0(0 \%)$ & $19(73.1 \%)$ & $0(0 \%)$ & $5(19.2 \%)$ \\
\hline Total & $13(8.7 \%)$ & $8(5.3 \%)$ & $67(44.7 \%)$ & $3(2.0 \%)$ & $52(34.7 \%)$
\end{tabular}




\section{Table 2}

Frequency of Specific Emotion Regulation Behaviors

\begin{tabular}{cccccc} 
Participant & $\begin{array}{c}\text { Pushing } \\
\text { Away }\end{array}$ & Digging In & Impulsivity & $\begin{array}{c}\text { Problem- } \\
\text { Solving }\end{array}$ & Acceptance \\
\hline 1 & $3(21.4 \%)$ & $8(57.1 \%)$ & $0(0 \%)$ & $3(21.4 \%)$ & $0(0 \%)$ \\
2 & $0(0 \%)$ & $13(61.9 \%)$ & $1(4.8 \%)$ & $5(23.8 \%)$ & $2(9.5 \%)$ \\
3 & $7(28.0 \%)$ & $15(60.0 \%)$ & $0(0 \%)$ & $1(4.0 \%)$ & $2(8.0 \%)$ \\
4 & $7(26.9 \%)$ & $7(26.9 \%)$ & $1(3.8 \%)$ & $5(19.2 \%)$ & $6(23.1 \%)$ \\
5 & $0(0 \%)$ & $0(0 \%)$ & $0(0 \%)$ & $2(100.0 \%)$ & $0(0 \%)$ \\
6 & $2(12.5 \%)$ & $1(6.3 \%)$ & $1(6.3 \%)$ & $5(31.3 \%)$ & $7(43.8 \%)$ \\
7 & $1(8.3 \%)$ & $1(8.3 \%)$ & $1(8.3 \%)$ & $3(25.0 \%)$ & $6(50.0 \%)$ \\
8 & $6(23.1 \%)$ & $9(34.6 \%)$ & $0(0 \%)$ & $10(38.5 \%)$ & $1(3.8 \%)$ \\
\hline Total & $26(18.3 \%)$ & $54(38.0 \%)$ & $4(2.8 \%)$ & $34(23.9 \%)$ & $24(16.9 \%)$
\end{tabular}


Table 3

Frequency of Specific Emotions and Emotion Regulation Behaviors

\begin{tabular}{lccccc} 
Behavior & Anger & Sadness & Anxiety & Guilt/Shame & Joy \\
\hline Pushing Away & $5(3.5 \%)$ & $3(2.1 \%)$ & $17(12.0 \%)$ & $1(0.7 \%)$ & $0(0 \%)$ \\
Digging In & $2(1.4 \%)$ & $1(0.7 \%)$ & $7(4.9 \%)$ & $0(0 \%)$ & $44(31.0 \%)$ \\
Impulsivity & $0(0 \%)$ & $0(0 \%)$ & $2(1.4 \%)$ & $0(0 \%)$ & $2(1.4 \%)$ \\
Problem-Solving & $1(0.7 \%)$ & $3(2.1 \%)$ & $26(18.3 \%)$ & $2(1.4 \%)$ & $2(1.4 \%)$ \\
Acceptance & $5(3.5 \%)$ & $1(0.7 \%)$ & $14(9.9 \%)$ & $0(0 \%)$ & $4(2.8 \%)$ \\
\hline Total & $13(9.2 \%)$ & $8(5.6 \%)$ & $66(46.5 \%)$ & $3(2.1 \%)$ & $52(36.6 \%)$
\end{tabular}


Figure 1

Emotional Intensity Within and Across Days for Each Participant

Participant 1, Day 1

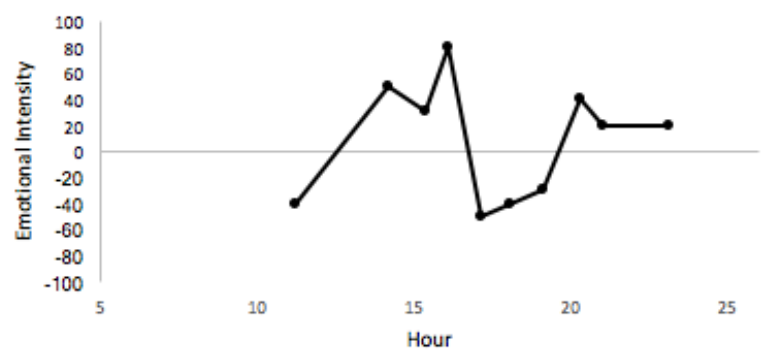

Participant 2, Day 1

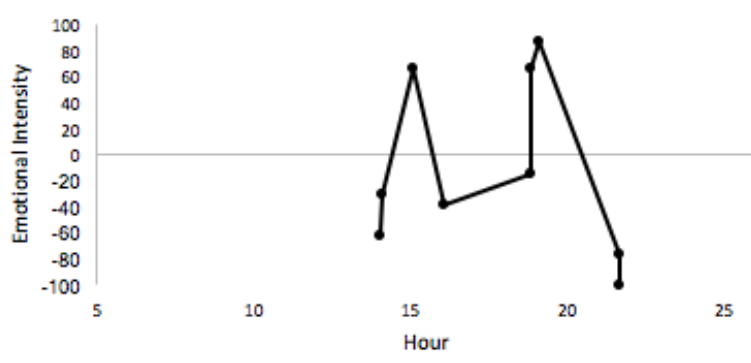

Participant 3, Day 1

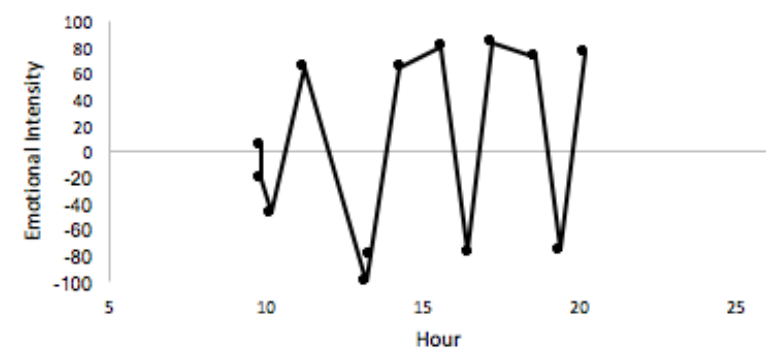

Participant 4, Day 1

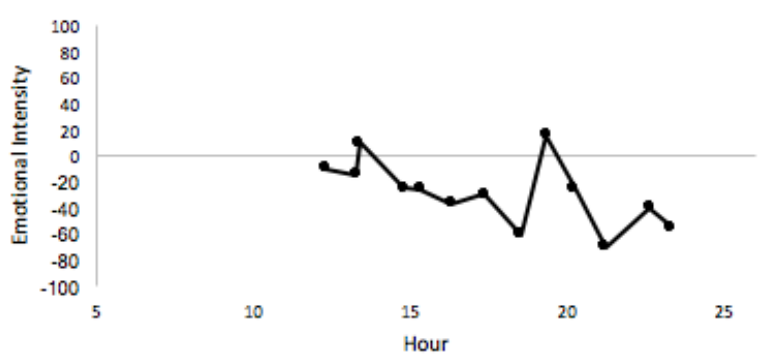

Participant 5, Day 1

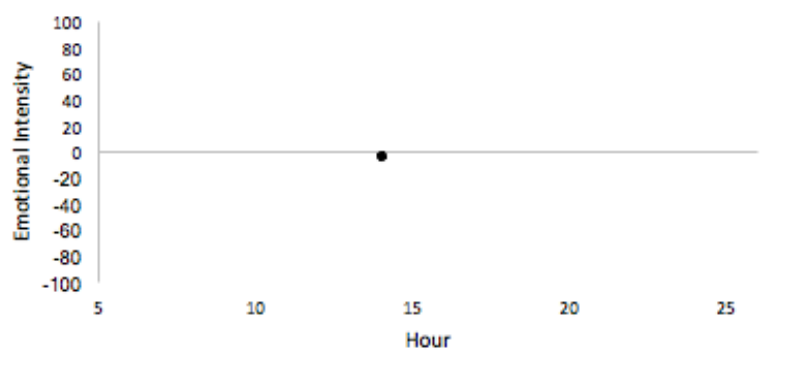

Participant 1, Day 2

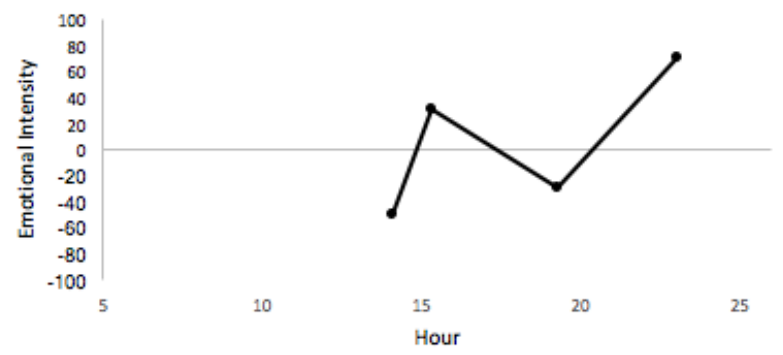

Participant 2, Day 2

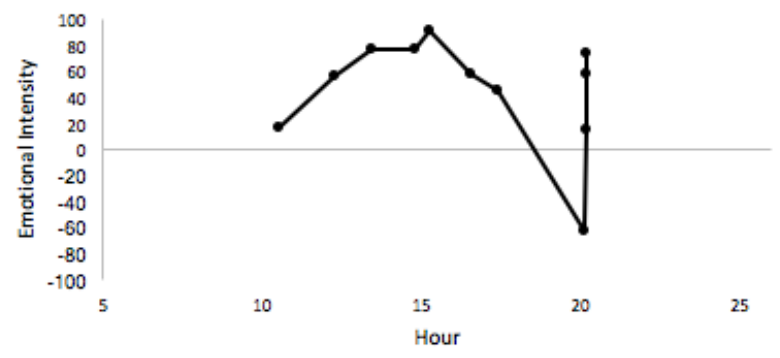

Participant 3, Day 2

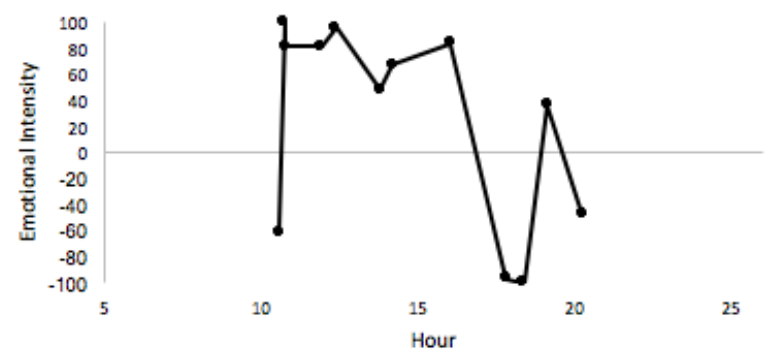

Participant 4, Day 2

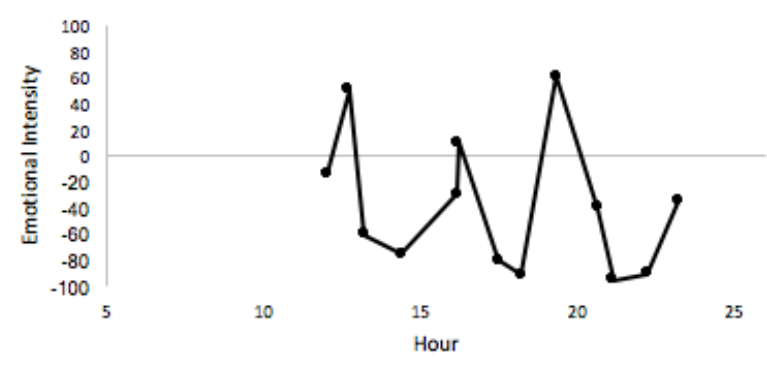

Participant 5, Day 2

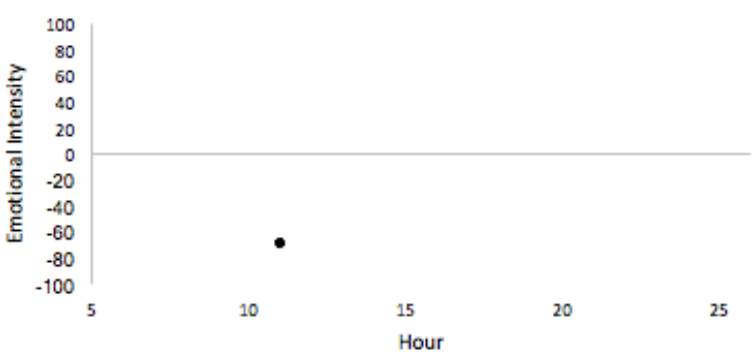


DAY IN THE LIFE OF BPD

36

Participant 6, Day 1

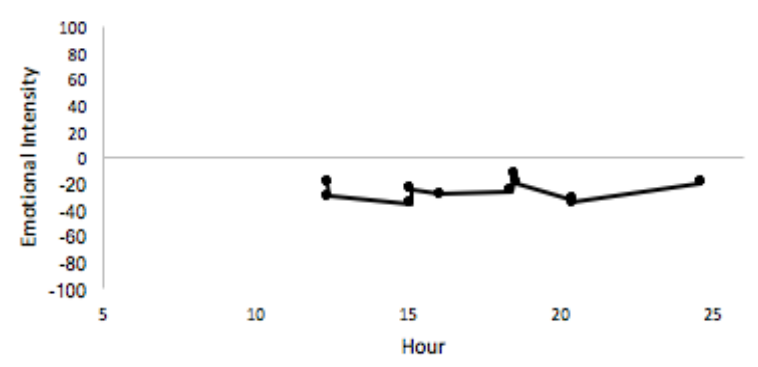

Participant 7, Day 1
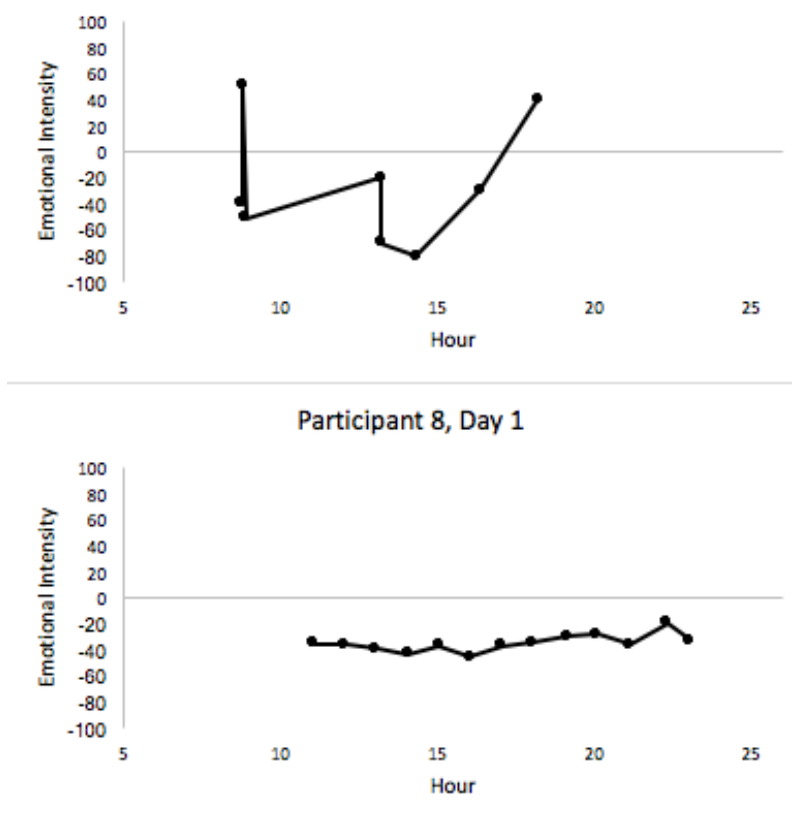

Participant 6, Day 2

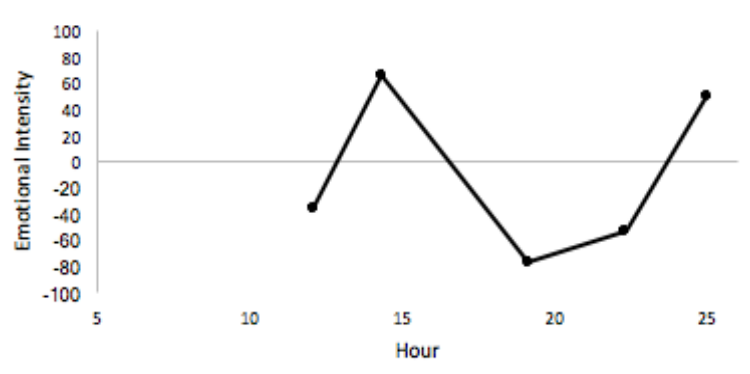

Participant 7, Day 2

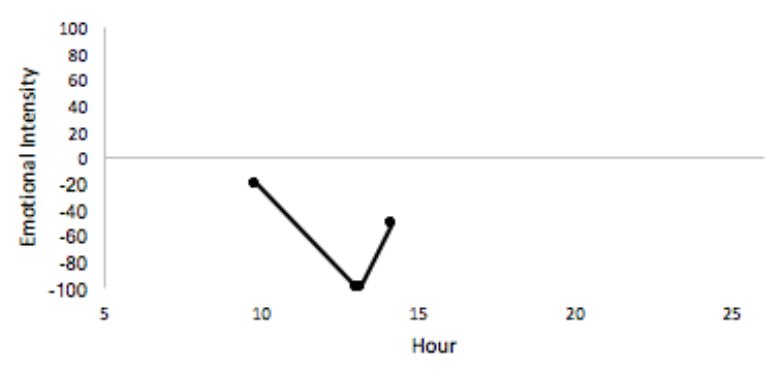

Participant 8, Day 2

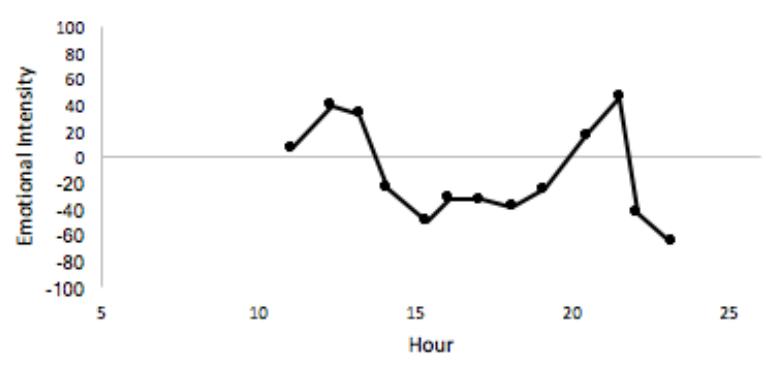




\section{Compliance with Ethical Standards}

- Disclosure of potential conflicts of interest: SSZ receives royalties from Oxford University Press in her role as an author of the treatment on which the intervention involved in the primary study is based. No other authors report any conflicts of interest.

- Research involving Human Participants and/or Animals: All study procedures were approved by the local university Institutional Review Board in accordance with the Declaration of Helsinki.

- Informed consent: All participants provided written informed consent before initiating study participation. 